\title{
Shear-induced interfacial sheath structure in isotactic polypropylene/glass fiber composites
}

\author{
Yijing Qin, Yahu Xu, Liying Zhang, Guoqiang Zheng*, Kun Dai, Chuntai Liu* \\ School of Materials Science and Engineering, The Key Laboratory of Material Processing and \\ Mold of Ministry of Education, Zhengzhou University, Zhengzhou, P.R. China \\ Xingru Yan, Jiang Guo, Zhanhu Guo* \\ Integrated Composites Laboratory (ICL), Department of Chemical \& Biomolecular \\ Engineering, University of Tennessee, Knoxville, TN 37996 USA
}

* Corresponding authors, Tel: +86 37163887600

E-mail: gqzheng@zzu.edu.cn (G. Zheng), ctliu@zzu.edu.cn (C. Liu), zguo10@utk.edu (Z. Guo) 


\begin{abstract}
Shear-induced interfacial sheath structure was generated by pulling a single glass fiber (GF) in the supercooled isotactic polypropylene (iPP) melt. Its morphological evolution and microstructure were investigated by means of polarized optical microscopy (POM), ex-situ polarized Fourier transform infrared spectroscopy (FTIR), ex-situ two-dimension wide-angle X-ray diffraction (2D-WAXD) and scanning electron microscopy (SEM). The results revealed that higher fiber-pulling speed was crucial to promote the formation of sheath structure. Such sheath structure was proved to be the most thermodynamically stable $\alpha$-crystal that could effectively induce the generation of $\beta$-cylindrite. Meanwhile, the thickness and orientation level of the sheath structure were increased with increasing the fiber-pulling speed. More interestingly, the sheath structure was composed of aligned shish-kebabs, and the lamellar-branched $\beta$-iPP crystals were directly "rooted" in these kebabs, viz., $\beta$-iPP crystals grew at the growth front of oriented fibrillar lamellae. This study on the interfacial sheath structure provides another direct evidence for the formation mechanism of $\beta$-iPP crystals upon shear field.
\end{abstract}

Keywords: $\beta$-cylindrite; sheath structure; shish-kebab. 


\section{Introduction}

Polymeric materials with a great potential to meet the requirements for desired applications have been widely used in the fields from daily packaging to aerospace. The physical and chemical properties of these polymers can be effectively tailored by controlling their structures at different scales [1]. In most polymer processing operations such as injection molding, extrusion molding, film blowing and fiber spinning, etc., the molten polymer chains are inevitably subjected to different degrees of shear flow field prior to crystallization. The shear flow produces chain orientation, and affects the crystallization kinetics as well as the crystal structure and morphology of the semicrystalline polymers [2-13]. Therefore, it is of great significance to obtain a full understanding of shear-induced crystallization, and the study on its mechanism has been an extremely active subject over the past few decades [8, 14-18].

Vast bodies of literatures have described the effects of flow on the crystallization of various polymers [19-21]. Among them, isotactic polypropylene (iPP) has been most frequently chosen as a model system owing to its (i) wide applications as a conventional plastic; (ii) lower nucleation density and crystal growth rate at relatively high temperatures, which enable the in-situ measurements; and (iii) diversified structures and morphologies that are sensitive to the changes in the processing parameters and molecular parameters, such as molecular weight, molecular weight distribution and chain branching etc [22-24]. Systematic rheo-optical and rheo-X-ray studies on the shear-induced crystallization of iPP indicate that the shear flow 
enhances the crystallization rate and promotes the formation of oriented structure, even extended chain shish structure $[6,19,23,25-35]$. Moreover, the crystallization from sheared or strained iPP melt was found to favor the formation of $\beta$-iPP crystals [8, 36-39]. However, the mechanism of the $\beta$-iPP crystals in flow field still remains a long-standing puzzle.

To explore the origin of the shear-induced $\beta$-iPP crystals, Varga et al. adopted an elegant experimental procedure by pulling glass fiber (GF) manually to simulate the shear field, and found that the $\beta$-rich cylindritic structure grew on a thin layer of oriented $\alpha$-iPP crystals along the GF surface $[8,14]$. They considered that the bundles of partially extended polymer molecular chains formed $\alpha$-row nuclei (i.e., shish structures) upon the shear field, and then the point-like $\beta$-crystal nuclei grew epitaxially onto these $\alpha$-row nuclei through $\alpha-\beta$ modification transition. Unfortunately, owing to the limited resolution of polarized optical microscopy (POM), the micromorphologic features of such thin layers of oriented $\alpha$-iPP crystals at the polymer/fiber interface cannot be captured. Li et al. claimed that the shear-induced ordered smectic domains rather than $\alpha$-row nuclei initiated the growth of $\beta$-iPP crystals [15]. However, further study by de Jeu et al. proved that the smectic ordering in the pre-sheared melt of iPP was not intrinsic to the system but instead resulted from the presence of small quantities of calcium stearate. In other words, there was no causal relation between the smectic ordering and the $\beta$-iPP crystals [16]. Subsequently, Yan et al. proposed that the formation of $\beta$-crystal nuclei might be restricted in a certain chain orientation window of the iPP melt, and the chain orientation outside of this 
window resulted in the formation of $\alpha$-iPP crystals. The formation of oriented $\alpha$-row nuclei was not considered to be a prerequisite for the formation of $\beta$-iPP crystals [17].

Our previous work on establishing an improved fiber-pulling device indicated that the interfacial crystallization kinetics was promoted by the presence of an interfacial shear stress, and there were two thresholds of the interfacial shear stress for two different crystal modifications (viz., $\alpha$ - and $\beta$-crystal) [18]. In this paper, an interesting coating structure around GF in the iPP/GF composite systems prepared by pulling a single GF in the supercooled iPP melt is reported and the coating structure is denominated as "sheath structure". To the best of the authors' knowledge, such structure is discovered for the first time in the iPP/GF composite systems. Furthermore, it can markedly induce the formation of $\beta$-cylindrite in the subsequent isothermal crystallization process. An attempt to estimate the molecular chains orientation level of the interfacial crystalline structure is made on the basis of the polarized Fourier transform infrared spectroscopy (FTIR) and two-dimension wide-angle X-ray diffraction (2D-WAXD) results. Finally, the microstructures of the sheath structure and $\beta$-crystal nucleation sites are further characterized by scanning electron microscopy (SEM). Accordingly, the relationship between the formation of $\beta$-iPP crystals and the oriented sheath structure is established.

\section{Experimental section}

\subsection{Materials}

The commercial iPP granules (T30S) were purchased from Lanzhou Petroleum Chemical Co., 
Ltd, with a melting flow index of $2.6 \mathrm{~g} / 10 \mathrm{~min}\left(190{ }^{\circ} \mathrm{C}\right.$ and $21.6 \mathrm{~N}$ load $), \bar{M}_{n}$ of $11 \times 10^{4} \mathrm{~g} / \mathrm{mol}$. The thin iPP films of $20-30 \mu \mathrm{m}$ in thickness were prepared by compression-molding the iPP granules at $200{ }^{\circ} \mathrm{C}$. The GFs utilized in this study were E-glass fibers with an average diameter of $14 \mu \mathrm{m}$, supplied by Zhengtong Chemical Industry Co., Ltd. The GFs were rinsed several times in acetone and deionized water successively with ultrasonication to remove the sizing agent, and dried in a vacuum oven at $80{ }^{\circ} \mathrm{C}$ for $10 \mathrm{~h}$.

\subsection{Experimental operation}

To study the shear-induced crystallization of iPP in the fiber-pulling system, a single GF was sandwiched between two pieces of compression-molded iPP thin films. As depicted in Fig. 1a, the sandwiched sample was first heated to $210{ }^{\circ} \mathrm{C}$ for $5 \mathrm{~min}$ to erase the possible effect of thermo-mechanical history on the subsequent crystallization, then cooled down to the fiber-pulling temperature, $T_{p}$ (set as $134{ }^{\circ} \mathrm{C}$ ), at a cooling rate of $20{ }^{\circ} \mathrm{C} / \mathrm{min}$. Once the temperature reached the $T_{p}$, the GF was pulled along the fiber axis by an improved fiber-pulling device at the preset fiber-pulling speed, $V_{p}$ (set as $0,10,50,90$ and $150 \mu \mathrm{m} / \mathrm{s}$ ). The fiber-pulling time, $t_{p}$, was $100 \mathrm{~s}$. Then the sample was crystallized isothermally for $20 \mathrm{~min}$ at $T_{c}=T_{p}=134^{\circ} \mathrm{C}$. Finally, the sample crystallized isothermally for 20 min was immediately removed from the hot stage and cryogenically quenched in an ice water mixture to reserve the resultant morphology for further FTIR, 2D-WAXD and SEM characterizations. For convenience, all the iPP/GF composite samples were labeled as PG-X, where $\mathrm{X}$ represented the fiber-pulling 
speed. For example, PG-10 represents the sample subjected to pre-shear at a fiber-pulling speed of $10 \mu \mathrm{m} / \mathrm{s}$.

(a)

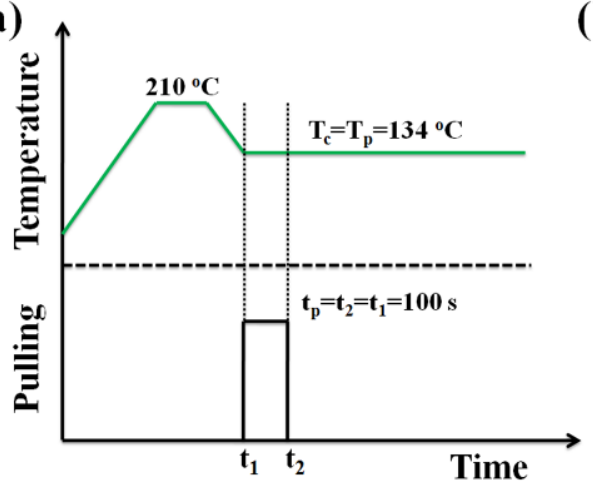

(b)

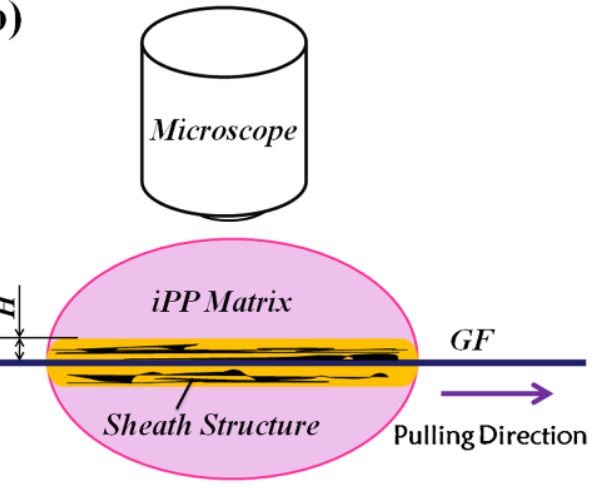

Fig. 1. Schematic representations of the (a) applied thermo-mechanical history and (b) shear-induced sheath structure around GF.

\subsection{Characterizations}

The morphological evolution of the sheath structure around GF during pulling the fiber and the subsequent isothermal crystallization was in-situ monitored by an Olympus BX51 POM, equipped with a Linkam THMS 600 hot-stage. The optical character, i.e., the sign of birefringence of the micromorphological feature could be determined by means of a primary red filter (U-TP530, $\lambda$-plate) located diagonally between the crossed polarizers. In this way, the first and third quarters of the sign were blue and the second and fourth ones were yellow when the spherulites were positive, while a reversed arrangement of the quarter was observed for negative spherulites.

The molecular chains orientation level of the interfacial crystalline structure was qualitatively investigated by a FTIR equipped with a polarizer (NICOLET 6700). The spectra were recorded from 400 to $4000 \mathrm{~cm}^{-1}$ with a resolution of $4 \mathrm{~cm}^{-1}$. 
To further characterize different crystal modifications and molecular chains orientation level in the interfacial crystalline structure, 2D-WAXD measurement was performed at the beam line BL16B1 at Shanghai Synchrotron Radiation Facility (SSRF). The radiation wavelength of X-ray was $0.124 \mathrm{~nm}$, and a two-dimensional detector MAR $165 \mathrm{CCD}(2048 \times 2048$ pixel with pixel size $80 \mu \mathrm{m})$ placed $118 \mathrm{~mm}$ away from the sample was used to collect 2D-WAXD patterns. The exposure time was $100 \mathrm{~s}$ for every 2D-WAXD image. All X-ray data were corrected for background (air and instrument) scattering before analysis.

It was worthwhile to point out that both FTIR and 2D-WAXD measurements were ex-situ carried out due to the restriction of the experimental devices and experimental conditions. All the samples were quenched within the ice water mixture after isothermal crystallization, thus the FTIR spectra and 2D-WAXD patterns represented the average intensities across the thickness of the final crystallized samples. Furthermore, in order to obtain the maximum diffraction intensity, rectangle segments with a width of $2 \mathrm{~mm}$ (including the total interfacial crystalline structures) were cut along the fiber axis from the quenched samples subjected to the same thermo-mechanical history, and then stacked orderly to attain a total thickness about $1 \mathrm{~mm}$.

To better characterize the supermolecular morphology at the lamellae level of the interfacial crystalline structure, SEM observation (JEOL JSM 7500F) was employed. The sample of PG-150 was chosen to be etched in a permanganate etching solution for $10 \mathrm{~h}$ approximately at room temperature. The etching solution was a mixture of $1: 1$ volume of concentrated nitrate and 
sulfuric acid by dissolving $0.7 \mathrm{wt} \%$ of potassium permanganate [40]. After etching, the sample was washed first with a mixture of sulfuric acid and water with a volume ratio of 2:7, and then rinsed with distilled water, hydrogen peroxide (30\%), and acetone several times, successively. The sample was finally sputter-coated with a thin gold layer after being dried in a vacuum oven for better imaging.

\section{Results and discussion}

\subsection{Morphology of the interfacial sheath structure}

A series of micrographs was taken during the process of pulling fiber to illustrate the evolution of interfacial morphology around GF, Fig. 2. Under a static condition, no crystal nuclei appear over the whole field of view in PG-0 at 100 s (see Fig. 2a), which substantially elucidates that the GF has no heterogeneous nucleation ability to the iPP in quiescent melt. With regard to the PG-10 (Fig. 2b), few spherulite nuclei are faintly observed at $100 \mathrm{~s}$ on the GF surface (shown by yellow arrows), but nothing is observed in the matrix far away from the fiber just like that of PG-0. This strongly confirms that even a slight shear can steadily facilitate the formation of crystal nuclei. Interestingly, as for the PG-50 (Fig. 2c), some fuzzy cotton-like coating begins to emerge around the GF when the fiber is pulled for $50 \mathrm{~s}$ (shown by white arrow), and the thickness of such coating accumulates gradually with the increasing pulling time. This phenomenon is extraordinarily distinct from that of PG-10. One should note that when the fiber-pulling speed is increased to 90 and $150 \mu \mathrm{m} / \mathrm{s}$ (see Fig. $2 \mathrm{~d}$ and e), such cotton-like coating 
begins to emerge even when the pulling time is as low as $25 \mathrm{~s}$ (shown by white arrows). It is worth mentioning that this interesting structure has never been reported in the iPP/GF composite systems in open literatures. Since its shape is analogous to a sheath, a "sheath structure" is used to denominate this structure.

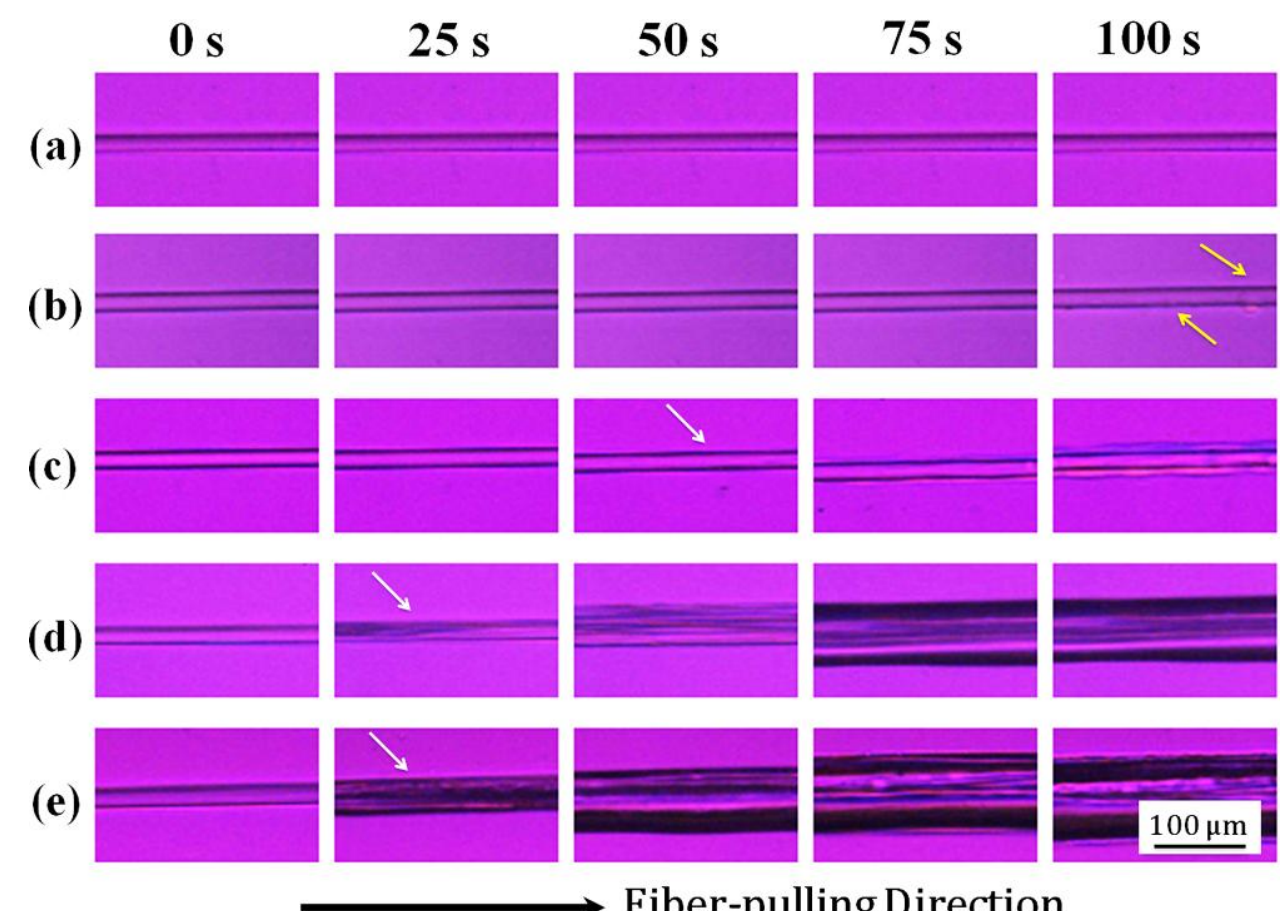

Fig. 2. Evolution of interfacial morphology around the GF during pulling fiber for various times: (a) PG-0, (b) PG-10, (c) PG-50, (d) PG-90 and (e) PG-150.

On the basis of the micrographs in Fig. 2, one can distinguish that such sheath structure surrounding GF is cylindrical, seeming to contain some oriented microstructures parallel to the GF axis. As a consequence, a schematic representation is depicted in Fig. 1b to better elucidate this interesting structure. Meanwhile, the thickness value of the sheath structure (shown by $H$ in Fig. 1b) unambiguously increases with the increasing fiber-pulling time and fiber-pulling speed, Fig. 2. In order to quantitatively manifest, the total diameter of the sheath structure $D_{1}$ 
(including GF) at $10 \mathrm{~s}$ intervals was measured with the assistance of Image-Pro Plus software, and thickness, $H$, was calculated according to Equation (1),

$$
H=\left(D_{1}-D_{2}\right) / 2
$$

where $H$ is the thickness of sheath structure, $D_{1}$ is the whole diameter of the sheath structure including GF, and $D_{2}$ is the average diameter of GF, i.e., $14 \mu \mathrm{m}$.
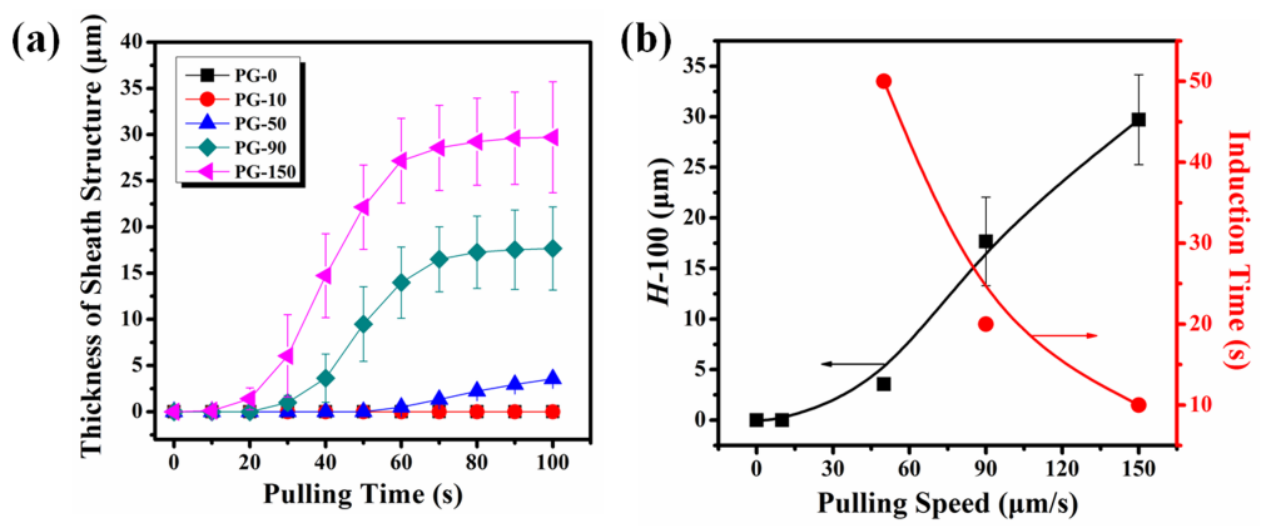

Fig. 3. (a) Time evolution profiles of the sheath structure's thickness during pulling fiber, and (b) variation tendency of the final thickness value of the sheath structure (denoted by $H-100$ ) and induction time as a function of the fiber-pulling speed.

Fig. 3a shows the calculated thickness of the sheath structure versus the fiber-pulling time at different fiber-pulling speeds. It demonstrates that when the fiber-pulling speed is lower than 10 $\mu \mathrm{m} / \mathrm{s}$, no visible sheath structure surrounding the GF surface is observed by POM. With the speed exceeding $50 \mu \mathrm{m} / \mathrm{s}$ (viz., 50, 90 and $150 \mu \mathrm{m} / \mathrm{s}$ ), there exists a short induction time for the generation of sheath structure, then the thickness value increases linearly and quickly, and it enhances gradually before reaching a plateau value.

To further understand the evolution of the sheath structure versus the pulling speed, the final thickness values of sheath structure ( $H-100)$, and the induction times for the occurrence of the 
sheath structure at different fiber-pulling speeds are potted in Fig. 3b. Here one can conclude that the $H-100$ for PG-0, PG-10, PG-50, PG-90 and PG-150 are about $0,0,3.6,17.7$ and $29.7 \mu \mathrm{m}$ respectively, manifesting that the final thickness value substantially is enhanced with the increasing fiber-pulling speed. With respect to the induction time, it is evident that a higher fiber-pulling speed is beneficial to the occurrence of sheath structure, and the induction time decreases sharply with the increasing fiber-pulling speed.

\subsection{Influence of interfacial sheath structure on crystalline morphology}

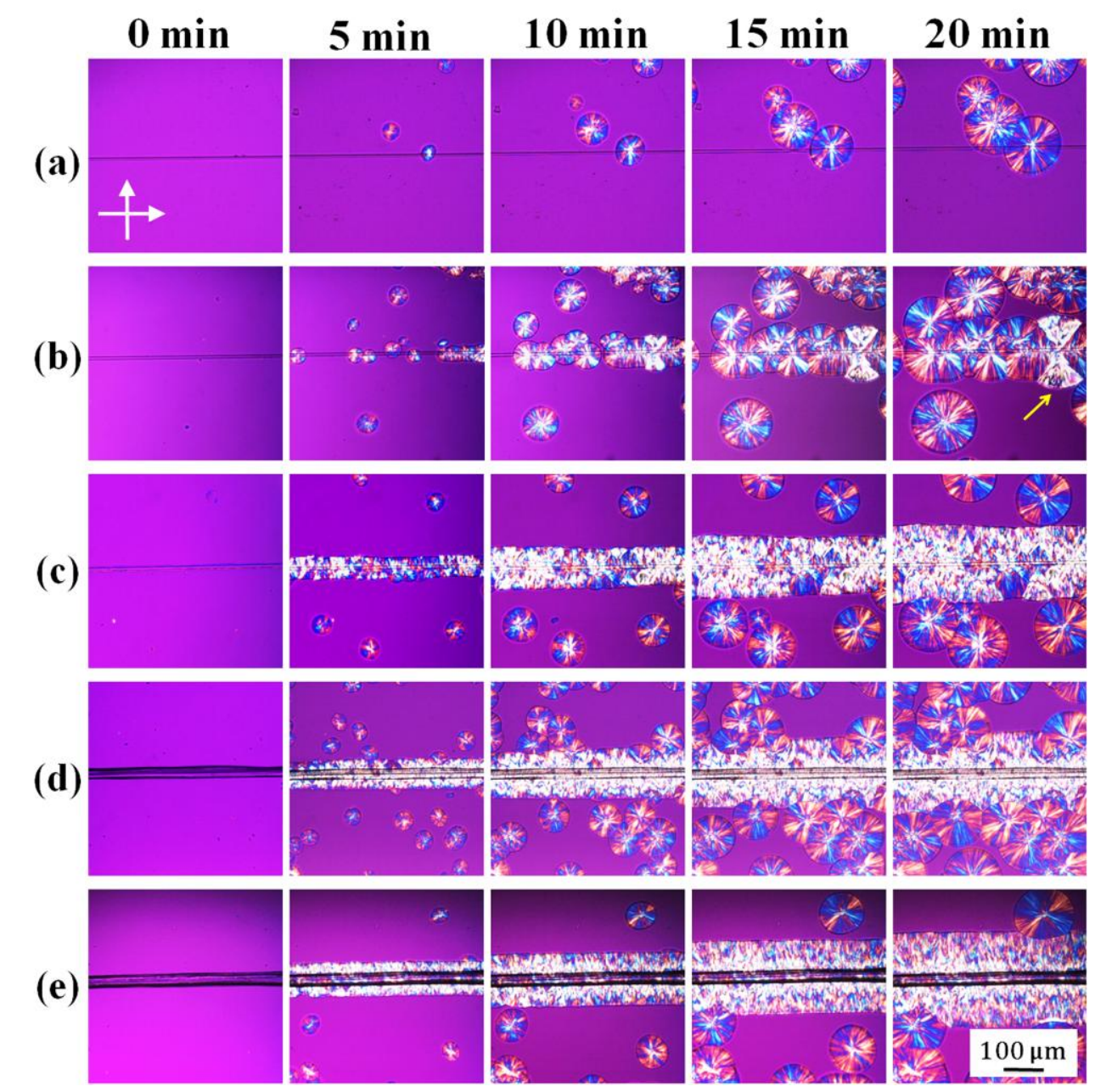

Fig. 4. Polarized optical micrographs of the interfacial crystalline morphology during isothermal crystallization at $134{ }^{\circ} \mathrm{C}$ for various times after shear cessation: (a) PG-0, (b) PG-10, (c) PG-50, (d) PG-90 and (e) PG-150. 
The white crossed arrows indicate the directions of polarizer and analyzer.

To investigate the influence of this sheath structure on the subsequent crystalline morphology, the samples were crystallized isothermally at $134{ }^{\circ} \mathrm{C}$ for $20 \mathrm{~min}$ after the pulling of the GFs was stopped, Fig. 4. One can observe that for the PG-0 (Fig. 4a), only mixed $\alpha$ spherulites, with irregularly alternating yellow and blue strips, evenly distributed with no trace of preferential nucleation close to the fiber. It clearly demonstrates that the pure GF has no iPP heterogeneous nucleation ability in the absence of shear. With regard to the PG-10 (Fig. 4b), a quasi-cylindritic structure comprised of $\alpha$ spherulites and few bright fan-shaped crystals (shown by yellow arrow) is recognized on the GF surface. These bright fan-shaped crystals are confirmed to be $\beta$-iPP crystals by both selective melting test at $158{ }^{\circ} \mathrm{C}[8,34]$ as displayed in Fig. $5 \mathrm{~b}$ and the WAXD measurement below (see Fig. 8b and Fig. 9a). The apparent increment of $\alpha$ spherulites around GF indicates that the interfacial nucleation of the iPP/GF composites can be remarkably promoted by the shear field. The PG-50 exhibits a typical mixed cylindrite outside the thin sheath structure, but this thin sheath structure cannot be detected any more after crystallizing isothermally for a period of time (see Fig. 4c). As shown in Fig. 5c, when the sample is heated to $158{ }^{\circ} \mathrm{C}$, only $\alpha$ spherulites in the matrix and the "saw-tooth" shaped $\alpha$-iPP crystals around the fiber are preserved. Regarding the PG-90 and PG-150 (Fig. 4d\&e), the interfacial cylindrites with strong birefringence are observed around the sheath structures. The selective melting test at $158{ }^{\circ} \mathrm{C}$ in Fig. 5d\&e well demonstrates that these bright cylindrites are predominately composed of $\beta$-iPP crystals, and moreover the sheath structures are more thermodynamically stable crystals than the 
$\beta$-iPP ones. The crystal modification of the sheath structure is deeply confirmed to be $\alpha$-crystal by the following 2D-WAXD measurement. In addition, the mixed $\alpha$ spherulites transform into negative ones exhibiting a clear Maltese cross at $158{ }^{\circ} \mathrm{C}$, which is consistent with previous observation [9].
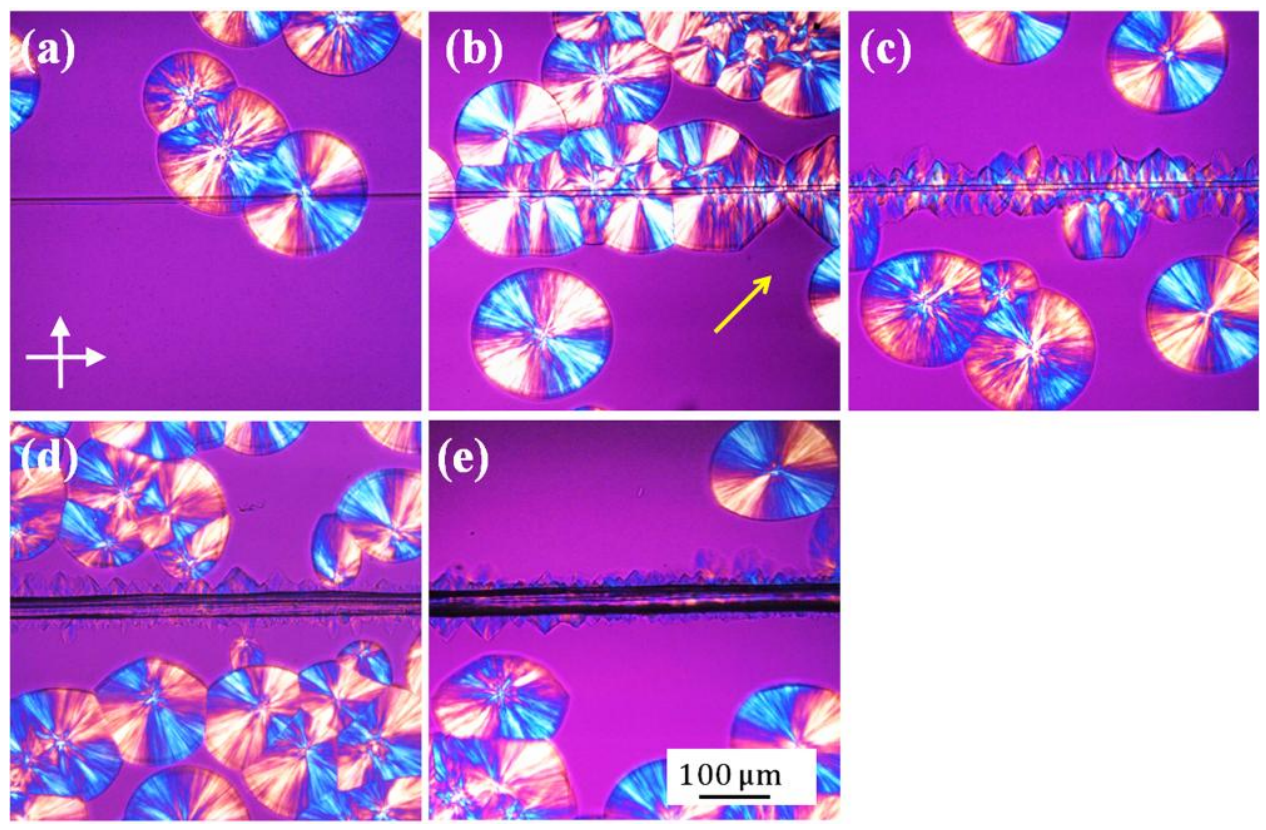

Fig. 5. Selective melting test of the $\beta$-iPP crystals at $158^{\circ} \mathrm{C}$ : (a) PG-0, (b) PG-10, (c) PG-50, (d) PG-90 and (e) PG-150. The white crossed arrows indicate the directions of polarizer and analyzer.

\subsection{Crystallization kinetics of different crystals}
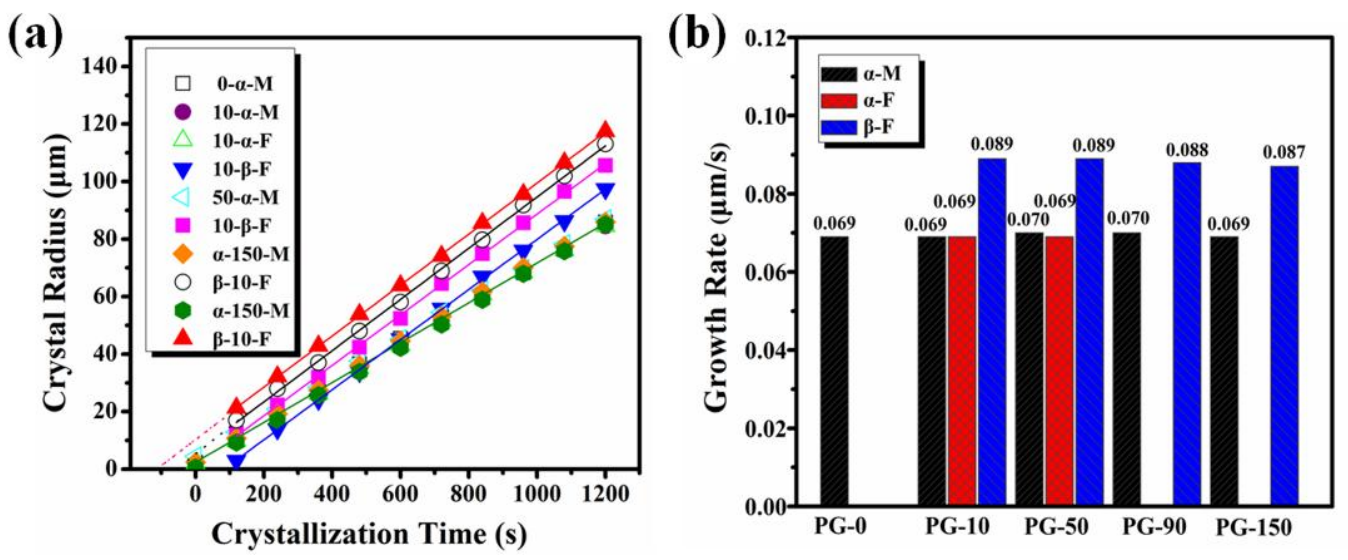

Fig. 6. (a) Variation of radii for the crystals with different crystal modifications versus crystallization time during the isothermal crystallization, and (b) growth rates of crystals. Here the numbers of $0,10,50,90$ and 
150 represent the fiber-pulling speeds, while $\alpha-\mathrm{M}$ represents the $\alpha$-iPP crystals in the iPP matrix and $\alpha / \beta-\mathrm{F}$ represents the $\alpha / \beta$-iPP crystals generated on the GF surface.

Herein, the crystallization kinetics of different crystals was obtained by the quantitative analysis. Fig. 6a displays the variation of the radii of different crystals isothermally crystallized versus crystallization time after pulling fibers at different speeds. Fig. $6 \mathrm{~b}$ presents the growth rates of crystals based on the linear fitting of the points in Fig. 6a. We can find that the growth rate of the $\beta$-iPP crystals is obviously higher than that of the $\alpha$-form ones, that is, they are respectively 0.089 and $0.069 \mu \mathrm{m} / \mathrm{s}$. It has been well documented that such issue is an intrinsic feature of the $\beta$-iPP crystals compared with its $\alpha$-form counterpart, namely, $\beta$-iPP crystals grow faster in the temperature range of $100-140{ }^{\circ} \mathrm{C}[8,17]$. In addition, it is clear that one specific crystal modification of the $\alpha$ - or $\beta$-crystal in matrix and those generated on GF surface has the same growth rate as illustrated in Fig. 6b. This is owing to the fact that the growth rate of a specific crystal modification in a given iPP based composite is mainly dependent on the crystallization temperature, while the imposed mechanical history prior to the crystallization has no significant influence on it [41]. Furthermore, one can obtain the crystallization induction periods from the intercept of the straight lines in Fig. 6a by extrapolation. However, as for the PG-90 and PG-150, the $\alpha$-iPP crystals on the GFs surface are fully covered by $\beta$-cylindrites, thus their crystallization induction periods cannot be acquired by extrapolation. Regardless of those aforementioned, it is interesting to find that in Fig. 6a, the crystallization induction periods of the $\alpha$-iPP crystals on the GF surface and in the matrix for PG-10 and PG-50 are almost identical, but 
severely different from that of the $\beta$-iPP crystals. One can draw a conclusion that a higher fiber-pulling speed leads to a shorter crystallization induction period of the $\beta$-iPP crystals. This discrepancy of the crystallization induction periods for two different crystal modifications may suggest that the formation of $\beta$-iPP crystals is more sensitive to a higher fiber-pulling speed than that of $\alpha$-form ones.

\subsection{Analysis for orientation level and crystal modification}
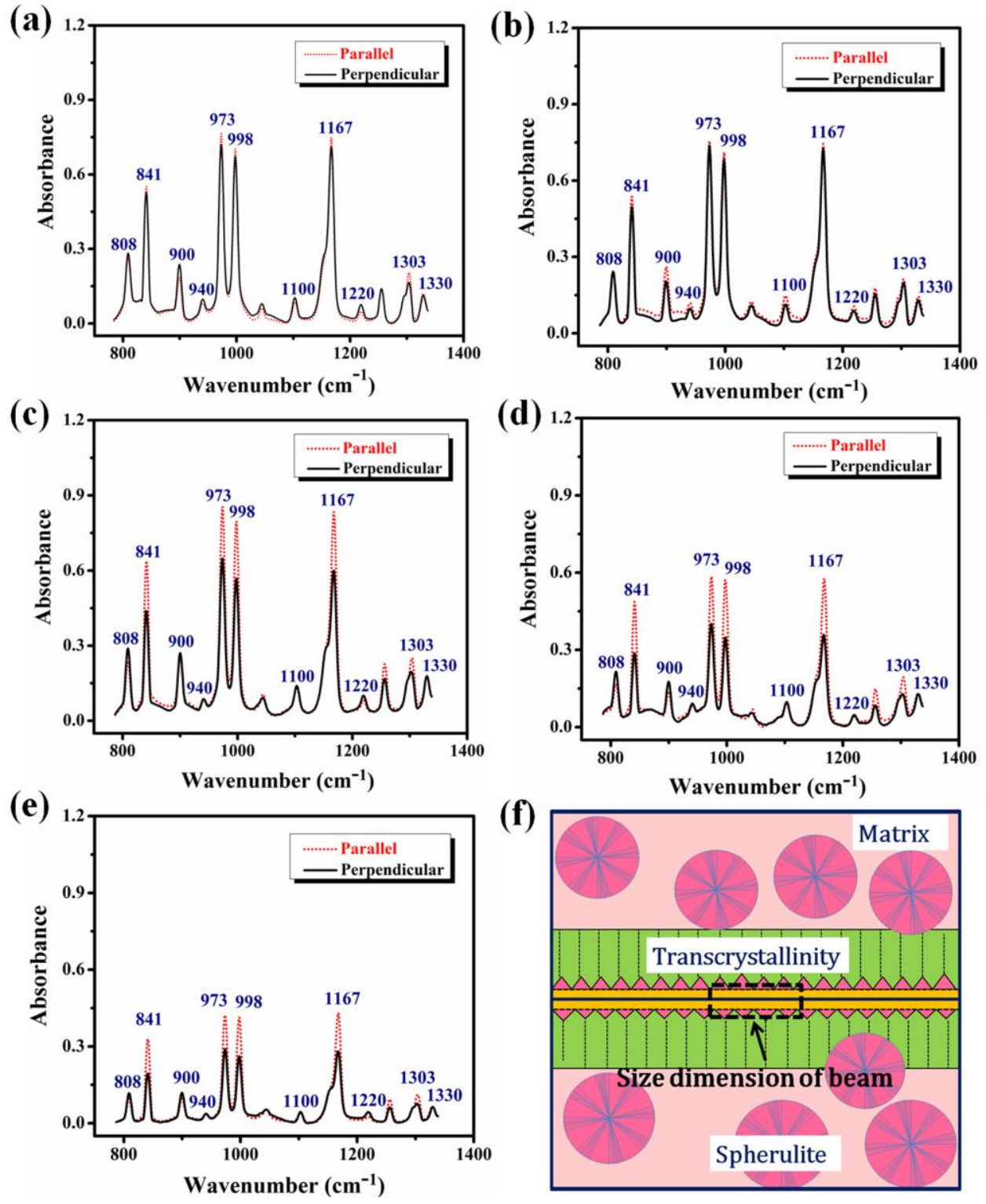
Fig.7. Polarized FTIR spectra of the interfacial crystalline structure close to GF of the resultant isothermally crystallized samples: (a) PG-0, (b) PG-10, (c) PG-50, (d) PG-90 and (e) PG-150. (f) Schematic diagram of the recorded region of the FTIR spectra (black-dotted rectangle).

To qualitatively study the effect of the fiber-pulling speed on the molecular chains orientation level in the interfacial crystalline structure close to the GF, the polarized FTIR test was carried out. The recorded region of FTIR spectra is illustrated by black-dotted rectangle in Fig. 7f. Fig. 7a-e show the polarized FTIR spectra of the PG-0, PG-10, PG-50, PG-90 and PG-150, respectively. Identical to the FTIR spectra of PG-0 (Fig. 7a), those of PG-10 (Fig. 7b) also have almost the same absorbance intensity in parallel- and perpendicular-polarized FTIR spectra. This suggests a poor molecular chains orientation level under lower fiber-pulling speed, or limited spatial resolution of this FTIR setup. As clearly depicted in Fig. 7c-e (on behalf of PG-50, PG-90 and PG-150), we can find notably different absorbance intensities in the parallel- and perpendicular-polarized FTIR spectra, that is, the intensities of the parallel-polarized FTIR spectra are dramatically stronger than that of the perpendicular-polarized FTIR spectra. This indicates that the molecular chains are principally oriented along the fiber axis (viz., fiber-pulling direction). Moreover, in order to further evaluate the orientation level of the interfacial crystalline structure, dichroic ratio was calculated using the following expression [42]:

$$
R=\frac{A_{/ l}}{A_{\perp}}
$$

where $R$ is dichroic ratio, $A_{/ /}$is the parallel-polarized infrared absorbance intensity and $A_{\perp}$ is the perpendicular-polarized one for a particular vibration in the observed FTIR spectra. In 
addition, if $A_{/ /}>A_{\perp}$, it is referred to as dichroic ratio of $\pi$, and this band is termed as parallel-band. On the contrary, if $A_{/ /}<A_{\perp}$, it is referred to as dichroic ratio of $\delta$, and this band is termed as perpendicular-band. For a polymer without any orientation, $R$ is always equal to 1 .

While it is a maximum value for a fully oriented polymer, and the maximum value changes with different bands.

Table 1 Dichroic ratios of different FTIR bands of the interfacial crystalline structure close to GF of resultant isothermally crystallized samples.

\begin{tabular}{cccccc}
\hline \multirow{2}{*}{ Wavenumber $\left(\mathrm{cm}^{-1}\right)$} & \multicolumn{5}{c}{ Dichroic ratio (R) } \\
\cline { 2 - 6 } & PG-0 & PG-10 & PG-50 & PG-90 & PG-150 \\
\hline 808 & 1.00 & 1.00 & 0.80 & 0.77 & 0.73 \\
841 & 1.00 & 1.01 & 1.48 & 1.71 & 1.79 \\
900 & 1.00 & 0.83 & 0.81 & 0.77 & 0.73 \\
940 & 1.00 & 0.91 & 0.89 & 0.71 & 0.59 \\
973 & 1.01 & 1.01 & 1.34 & 1.47 & 1.49 \\
998 & 1.01 & 1.02 & 1.44 & 1.68 & 1.70 \\
1100 & 1.00 & 0.74 & 0.87 & 0.87 & 0.88 \\
1167 & 1.00 & 1.01 & 1.40 & 1.58 & 1.61 \\
1220 & 0.99 & 0.81 & 0.73 & 0.59 & 0.68 \\
1303 & 1.00 & 1.01 & 1.27 & 1.51 & 1.52 \\
1330 & 0.98 & 0.99 & 0.89 & 0.90 & 0.87 \\
\hline
\end{tabular}

The dichroic ratios of different FTIR bands for all the samples are summarized in Table 1. Apparently, one can find that regardless of the parallel- and perpendicular-band, all the values of $R$ for PG-0 and PG-10 are very close to 1 , and $R$ for PG-50 just has little departure from 1. However, $R$ for PG-90 and PG-150 obviously diverges from 1 compared with other samples. These dichroic ratios further verify that the higher the fiber-pulling speed is, the more remarkable 
extent of the molecular chains orientation in interfacial crystalline structure can be produced.

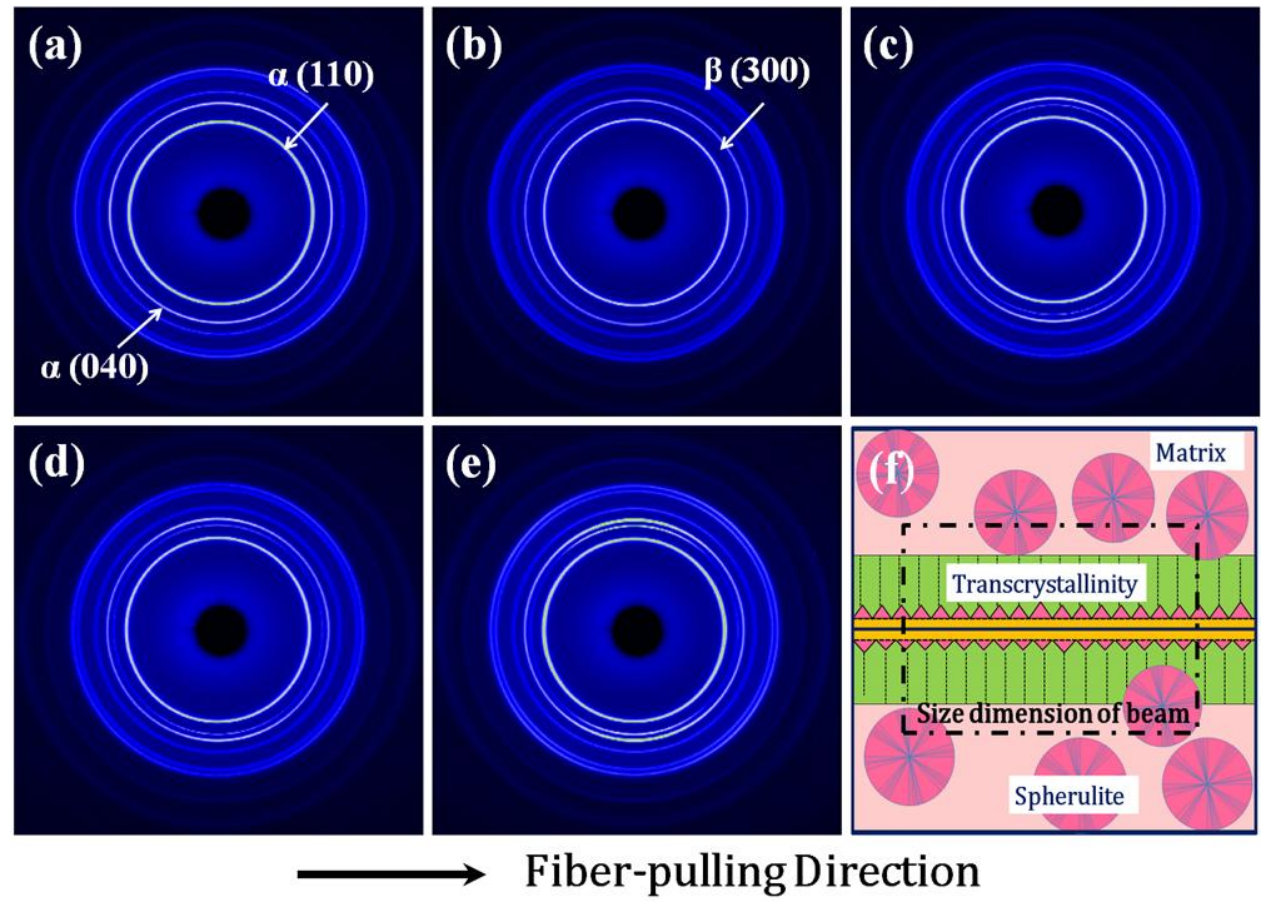

Fig. 8. 2D-WAXD patterns of the resultant isothermally crystallized samples: (a) PG-0, (b) PG-10, (c) PG-50, (d) PG-90 and (e) PG-150. (f) Schematic diagram of the detected region of 2D-WAXD beam (black-dotted rectangle).

The 2D-WAXD measurement was used to further characterize the interfacial crystalline structure of different samples subjected to various shear histories. The detected region of the 2D-WAXD beam is depicted by black-dotted rectangle in Fig. 8f, and the representative 2D-WAXD patterns of all the samples are summarized in Fig. 8a-e. One can notice that for the sample of PG-0 (Fig. 8a), the X-ray diffraction pattern presents diffusing rings, suggesting no orientation in the sample under a static condition. These reflections from inner to outer can be indexed as (110), (040), (130), (111) and (-131) or (041) lattice planes of $\alpha$-monoclinic form of iPP [43]. While for the samples subjected to shear prior to the isothermal crystallization as displayed in Fig. 8b-e, the diffusing ring of $\alpha(040)$ lattice plane can be distinguished into two 
diffraction arcs in the meridional direction, directly indicating the occurrence of oriented $\alpha$-iPP crystals. Moreover, additional indistinct six-arcs pattern of $\beta(300)$ diffraction appears between $\alpha(110)$ and $\alpha(040)$, suggesting that the parent-daughter lamellar branching structure of the $\beta$-iPP crystals is formed according to our previous study $[44,45]$. This lamellar branching structure in $\beta$-iPP crystals is further confirmed by the following SEM observation.
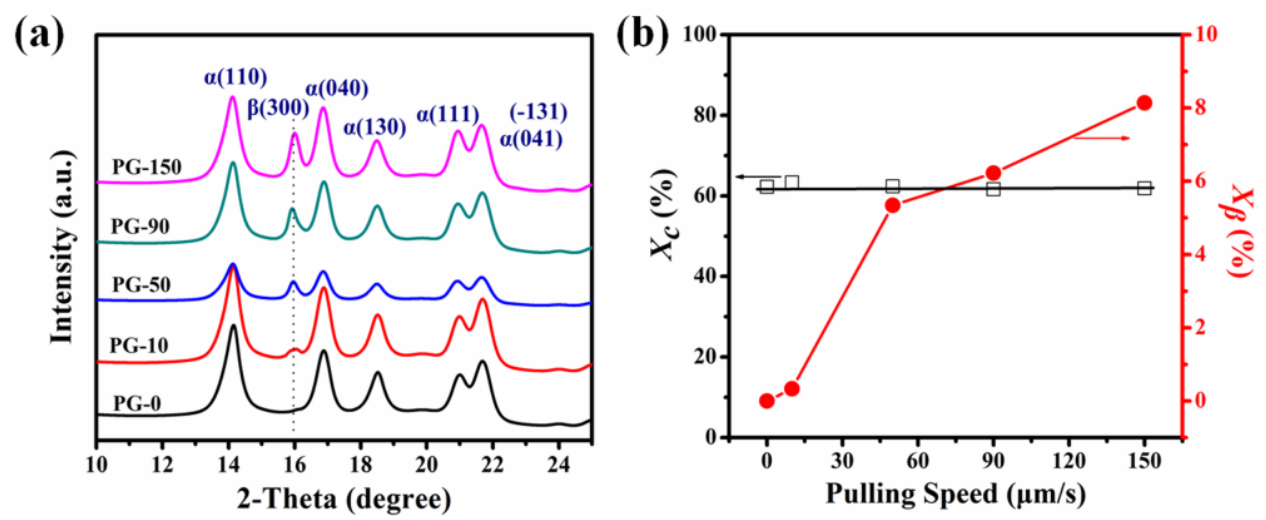

Fig. 9. (a) Selected one-dimensional WAXD profiles of the resultant isothermally crystallized samples extracted from the corresponding 2D-WAXD patterns, and (b) overall crystallinity $\left(X_{c}\right)$ as well as crystallinity of $\beta$-iPP crystals $\left(X_{\beta}\right.$ ) versus the fiber-pulling speed.

In order to quantify the crystallinity of $\beta$-iPP crystals $\left(X_{\beta}\right)$ as a function of the fiber-pulling speed, one-dimensional WAXD profiles are illustrated in Fig. 9a, where a peak at around $2 \theta=16^{\circ}$ is ascribed to the (300) lattice plane of $\beta$-form. Firstly, the overall crystallinity $\left(X_{c}\right)$ was calculated by Equation (3),

$$
X_{c}=\left(1-\frac{A_{\text {amorp }}}{\sum A_{\text {cryst }}+A_{\text {amorp }}}\right) \times 100 \%
$$

where $A_{\text {amorp }}$ and $A_{\text {cryst }}$ are the fitted areas of the amorphous and crystalline peaks, 
respectively. The relative content of $\beta$-iPP crystals $\left(K_{\beta}\right)$ can be evaluated by Turner-Jones criterion via Equation (4) [46],

$$
K_{\beta}=\frac{A_{\beta(300)}}{A_{\alpha(110)}+A_{\alpha(040)}+A_{\alpha(130)}+A_{\beta(300)}}
$$

where $A_{\beta(300)}$ is the fitted area of the (300) reflection peak, $A_{\alpha(110)}, A_{\alpha(040)}$ and $A_{\alpha(130)}$ are the areas of the $(110)_{\alpha},(040)_{\alpha}$ and $(130)_{\alpha}$ reflection peaks, respectively. Meanwhile, the crystallinity of $\beta$-iPP crystals $\left(X_{\beta}\right.$ ) is given by Equation (5).

$$
X_{\beta}=K_{\beta} \times X_{c}
$$

The $X_{c}$ and $X_{\beta}$ as a function of the fiber-pulling speed are plotted in Fig. 9b. The $X_{c}$ of all the samples is observed to nearly maintain a plateau ( $c a .62 \%)$. This should be attributed to their same crystallization temperature and crystallization time. Furthermore, it is outstanding that the $X_{\beta}$ is enhanced with the increasing fiber-pulling speed, well consistent with the optical microscopy observation, Fig. 4.
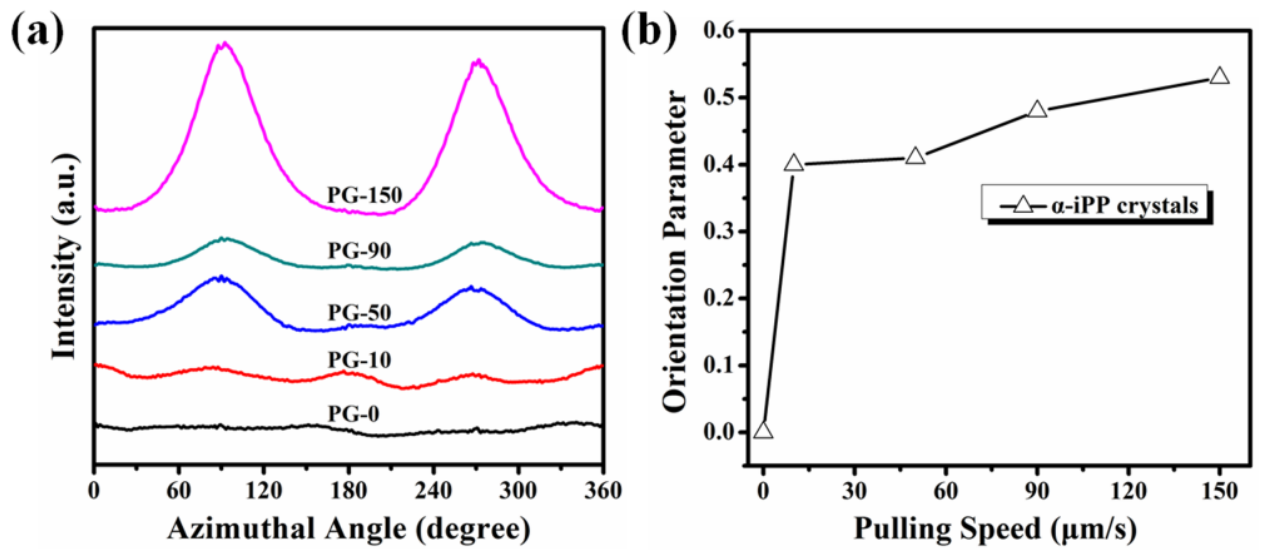

Fig.10. (a) Azimuthal intensity distributions of (040) lattice plane extracted from corrected 2D-WAXD patterns, and (b) orientation parameter of $\alpha$-iPP crystals versus the fiber-pulling speed.

In order to clearly quantify the orientation level of the $\alpha$-iPP crystals in the interfacial 
crystalline structure, the azimuthal intensity distributions of (040) lattice plane at $2 \theta=16.9^{\circ}$ deriving from corrected 2D-WAXD patterns are depicted in Fig. 10a. Compared with the static sample of PG-0, the (040) diffraction of PG-50, PG-90 and PG-150 show peaks at azimuthal angles of $90^{\circ}$ and $270^{\circ}$, indicating that the polymer chains in the $\alpha$-iPP crystals are oriented along the fiber-pulling direction. While for PG-10, the (040) diffraction shows peaks at $0^{\circ}, 90^{\circ}$, $180^{\circ}$ and $270^{\circ}$, indicating that the polymer chains are not parallel to the fiber-pulling direction strictly. Simultaneously, it is observed that the (040) diffraction in PG-90 and PG-150 have prominent narrower azimuthal width in the meridional direction than that in PG-50. Nevertheless, there is $\beta$-cylindrite rather than $\alpha$-cylindrite in the samples of PG-90 and PG-150. Taking the detected region into account, the appearance of narrower azimuthal width in (040) diffraction in PG-90 and PG-150 should be mainly attributed to the interfacial sheath structures. In view of this, one can further exactly conclude that: (i) the crystal modification of sheath structure is $\alpha$-crystal, as deduced from the selective melting test of $\beta$-iPP crystals in Fig. 5; and (ii) the interfacial sheath structure is well oriented along the fiber-pulling direction, which is consistent with the FTIR test in Fig. 7.

Then the level of orientation of crystals at the interface was calculated by the Herman's orientation parameter $f$ defined as Equation (6) [47],

$$
f=\frac{3<\overline{\cos ^{2} \phi}>-1}{2}
$$

where the angle of $\phi$ denotes the angle between the axis of interest and the unique axis. The 
fiber-pulling direction is chosen as the reference axis to calculate the orientation parameter by the normal vector of the lattice plane. Hence, $\left\langle\overline{\cos ^{2} \phi}\right\rangle$ can be obtained from azimuthal scattering intensity distribution by Equation (7),

$$
<\overline{\cos ^{2} \phi}>=\frac{\int_{0}^{\pi / 2} I(\phi) \sin \phi \cos ^{2} \phi d \phi}{\int_{0}^{\pi / 2} I(\phi) \sin \phi d \phi}
$$

where $I(\phi)$ is the scattering intensity along the angle of $\phi$. The orientation parameter attains a value of unity when all the crystals are oriented with their interested axis parallel to the reference direction (i.e., the fiber-pulling direction), a value of -0.5 corresponds to a state that all the interested axis are perpendicular to the reference direction, while totally random orientation gives a value of 0 [48].

The detailed orientation parameter of the $\alpha$-iPP crystals as a function of the fiber-pulling speed is plotted in Fig. 10b. The PG-0 is observed to exhibit no preferred orientation, which can be interpreted from the polarized optical micrographs where only some $\alpha$ spherulites are randomly distributed in the matrix, Fig. 4a. For PG-10 and PG-50, they have almost the same orientation parameters, denoting that their $\alpha$-cylindrites have analogous morphology, and the effect of thin oriented sheath structure for PG-50 is negligible. Comparatively, the orientation parameters increase progressively for PG-90 and PG-150, which are evidently larger than those of PG-10 and PG-50. These results straightly elucidate that the sheath structure has more notable orientation level than that of $\alpha$-cylindrite. 


\subsection{SEM observation}

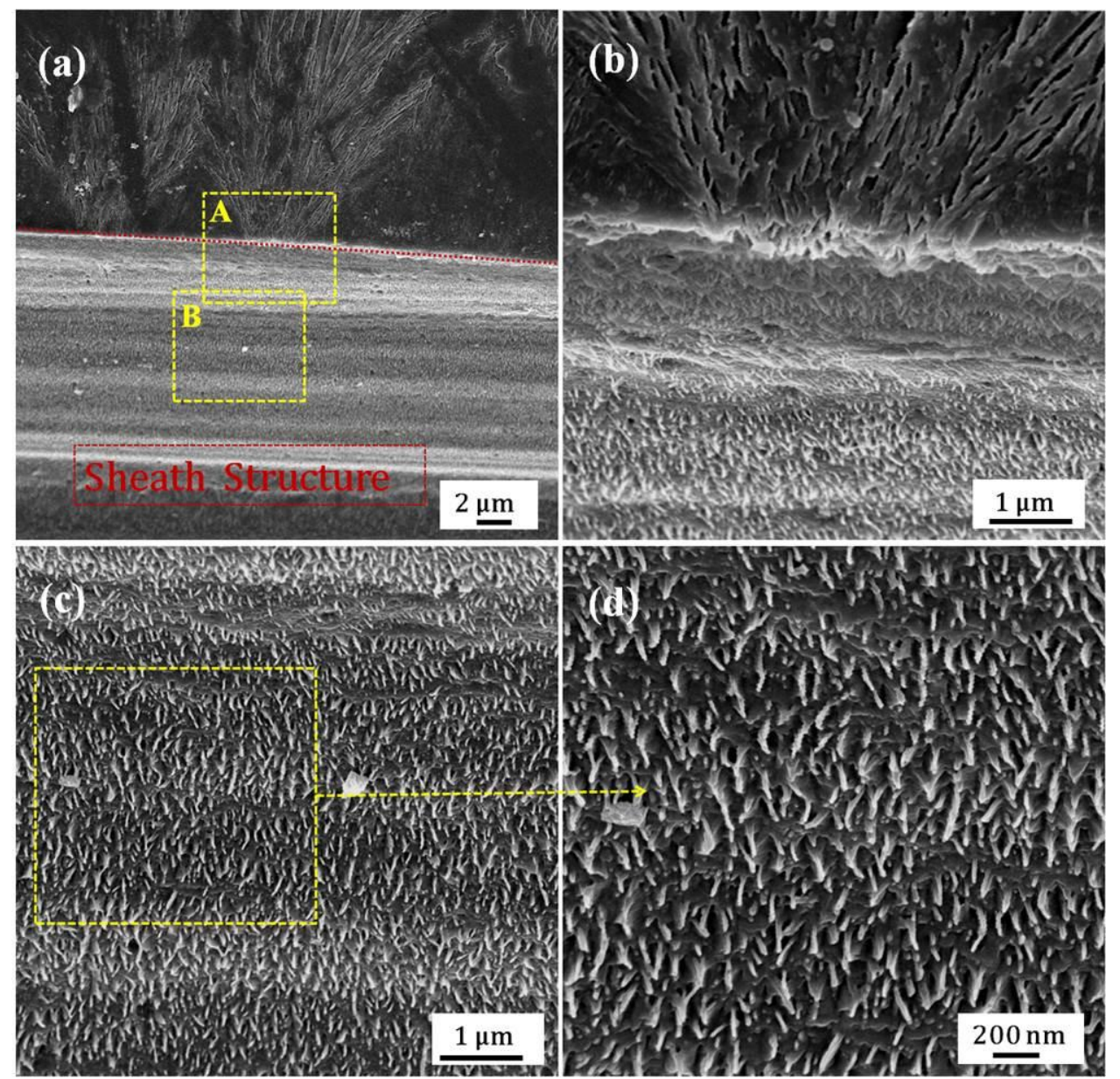

Fig. 11. SEM images of (a) an overview of the shear-induced $\beta$-cylindrite in PG-150, (b) the primary nucleation sites of the $\beta$-iPP crystals, and (c, d) the top surface of sheath structure. (a) The boundary between the $\beta$-cylindrite and sheath structure is traced with the dotted red line; (b) local higher magnification image of primary nucleation sites of $\beta$-iPP crystals in the yellow rectangle A of (a); (c) local higher magnification image in the yellow rectangle B of (a); (d) local higher magnification image of (c) showing the oriented fibrillar lamellae at the top surface of sheath structure.

To further explore the microstructures developed in the interfacial sheath structure and the relationship between the primary nucleation sites of the $\beta$-iPP crystals and the sheath structure, the SEM observation at a smaller scale was carried out. It should be noted that, in order to obtain more insight into the microstructures, the sample of PG-150 with the maximal thickness of the 
sheath structure was chosen. As depicted in Fig. 11a, one can find that the fan-shaped $\beta$-iPP crystals grow directly on the surface of the sheath structure. The close-up of the primary nucleation sites of the $\beta$-iPP crystals in Fig. 11b explicitly reveals that a cluster of edge-on lamellae of the $\beta$-iPP crystals well "root" in the sheath structure, that is, the $\beta$-crystal nuclei are embedding into the sheath structure. Moreover, it is well demonstrated that the lamellar branching occurs in the $\beta$-iPP crystals, and the subsequent proliferating results in the fan-shaped crystalline morphology. This is well consistent with the aforementioned six-arcs pattern of (300) diffraction. From the local higher magnification images at the top surface of sheath structure (see Fig. 11c and d), lots of densely aligned fibrillar $\alpha$-iPP lamellae are observed to grow outwards, and their orientation direction is perpendicular to the fiber axis. Herein, the nucleation and growth of the lamellae normal to the flow direction are noted to be impossible if there are no shish structures [6]. Therefore, one can deduce that the shish structures should exist in the sheath structure, but it is difficult to be observed by SEM. The invisible shish structures can be reasonably attributed to their tiny size and the fact that they are covered by the dense fibrillar lamellae.

Based on the above findings, the development of the shear-induced sheath structure and $\beta$-iPP crystals is schematically depicted in Fig. 12. In the sheared supercooled iPP melt by pulling fiber, the bundles of the partially extended polymer molecular chains close to the fiber surface form shish structures with tiny size, and they later give rise to the epitaxial growth of the fibrillar 
lamellae. In other words, the sheath structure is composed of large numbers of aligned shish-kebabs. Under suitable experimental conditions (widely accepted theory is kinetic prerequisite of the $\alpha-\beta$ modification transition), the $\beta$-crystal nuclei form at the growth front of the aligned $\alpha$-crystal lamellae. However, Varga et al. believed that the linear $\alpha$-row nuclei (i.e., shish structure) directly induced the epitaxial growth of the point-like $\beta$-crystal nuclei through the $\alpha-\beta$ modification transition $[8,14]$, which are different from our observed results. Moreover, in our current work, no direct experimental evidence can be obtained to support the existence of smectic ordering put forward by $\mathrm{Li}$ et al [15]. It is noteworthy that in our experiment, the $\beta$-iPP crystals generate only after the appearance of oriented sheath structure (viz., oriented $\alpha$-iPP crystals) during the isothermal crystallization, as proved by the selective melting test of the $\beta$-iPP crystals and SEM observation. Therefore, the concept of chain orientation window proposed by Yan et al. [17] seems also inconsistent with our results. Combining the above discussion, the interfacial sheath structure is a new finding in the field of shear-induced crystallization, and its appearance has given a new insight into the formation mechanism of $\beta$-iPP crystals in shear flow field. As for the inner microstructure of sheath structure, future research is required. 


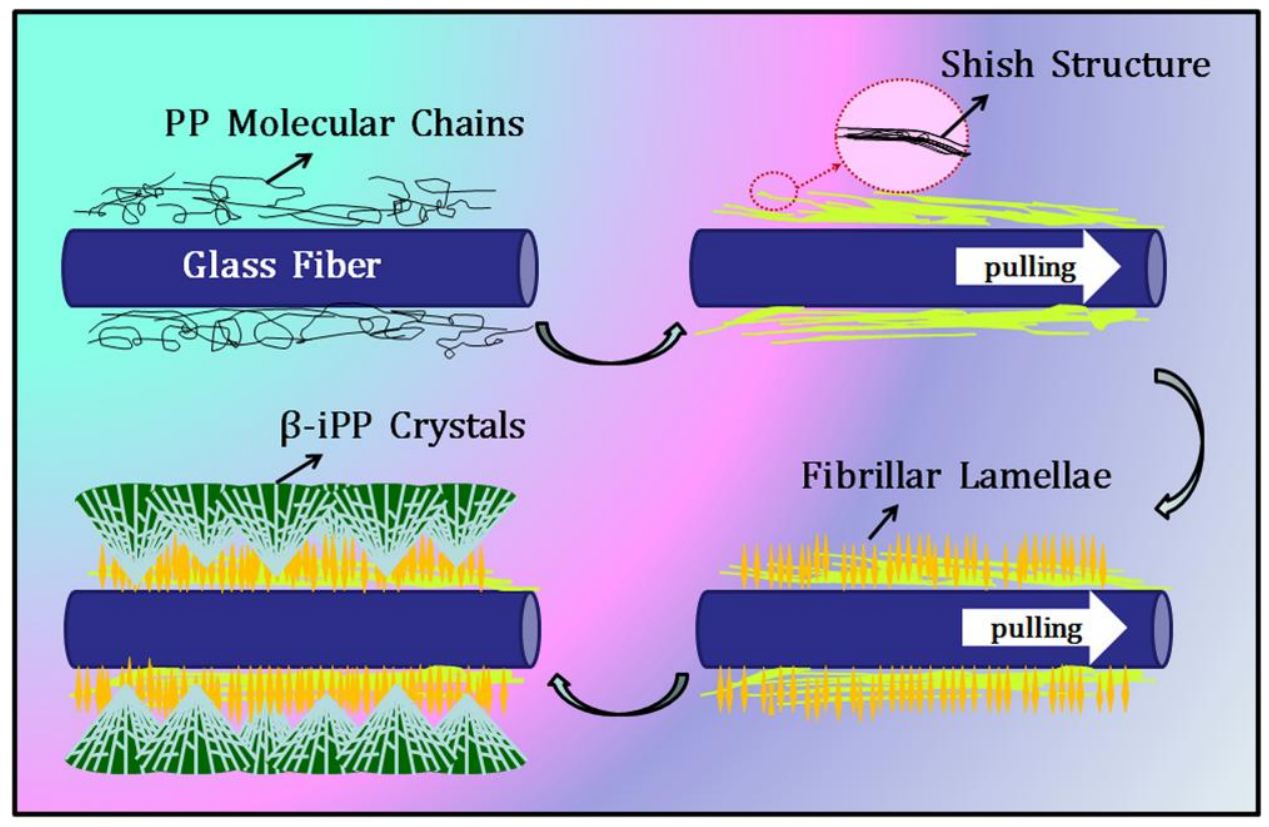

Fig. 12. Schematic diagram for the formation mechanism of the shear-induced sheath structure and $\beta$-iPP crystals.

\section{Conclusions}

The shear-induced interfacial sheath structure in the iPP/GF composites produced by pulling fiber has been studied by POM, as well as ex-situ FTIR, 2D-WAXD and SEM. The results suggest that a higher fiber-pulling speed is crucial to favor the formation of sheath structure, and its thickness increases with the increasing fiber-pulling time. Furthermore, its final thickness remarkably is increased with the increasing fiber-pulling speed. On the contrary, its induction time sharply reduces with the increasing fiber-pulling speed. The crystal modification of sheath structure is the most thermodynamically stable $\alpha$-crystal, and it can prominently induce the formation of $\beta$-cylindrite. As evidenced by the FTIR, 2D-WAXD data, the molecular chains of the sheath structure are well oriented along the fiber-pulling direction, and the orientation parameter is enhanced with the increasing fiber-pulling speed. More interestingly, the 
microstructure of the sheath structure turns out to be aligned shish-kebabs composed of the shish structures and epitaxial fibrillar lamellae, and afterwards the lamellar-branched $\beta$-iPP crystals directly grow at the growth front of these oriented fibrillar lamellae. Clearly, the origin of the $\beta$-iPP crystals subjected to the shear flow field in this study is different from those previously proposed.

\section{Acknowledgments}

The financial supports from National Natural Science Foundation of China $(51173171,11172271$, and 11432003), HASTIT and Plan for Scientific Innovation Talent of Henan Province for

financial support are appreciated. Z. Guo appreciates the support from National Science Foundation (NSF, CMMI 13-14486) USA.

\section{References}

[1] Liu Q, Sun X, Li H, Yan S. Polymer 2013; 54(17): 4404-4421.

[2] Jay F, Haudin JM, Monasse B. Journal of Materials Science 1999; 34(9): 2089-2102.

[3] Eder G, Janeschitz-Kriegl H. Materials science and technology 1997.

[4] Duplay C, Monasse B, Haudin JM, Costa JL. Journal of Materials Science 2000; 35(24): 6093-6103.

[5] Abuzaina FM, Fitz BD, Andjelić S, Jamiolkowski DD. Polymer 2002; 43(17): 4699-4708.

[6] Kumaraswamy G, Issaian AM, Kornfield JA. Macromolecules 1999; 32(22): 7537-7547.

[7] Varga J. Die Angewandte Makromolekulare Chemie 1983; 112(1): 191-203.

[8] Varga J, Karger-Kocsis J. Journal of Polymer Science Part B: Polymer Physics 1996; 34(4): 657-670. 
[9] Wu C-M, Chen M, Karger-Kocsis J. Polymer 1999; 40(15): 4195-4203.

[10] Gahleitner M, Wolfschwenger J, Bachner C, Bernreitner K, Neißl W. Journal of Applied Polymer Science 1996; 61(4): 649-657.

[11] Huang HX. Journal of Applied Polymer Science 1998; 67(12): 2111-2118.

[12] Kalay G, Bevis MJ. Journal of Polymer Science Part B: Polymer Physics 1997; 35(2): 265-291.

[13] Ryan AJ. Polymer processing and structure development: Dodrecht: Academic Publishers, 1998.

[14] Varga J, Karger-Kocsis J. Composites science and technology 1993; 48(1): 191-198.

[15] Li L, de Jeu WH. Faraday Discussions 2005; 128(0): 299-319.

[16] Byelov D, Panine P, de Jeu WH. Macromolecules 2007; 40(2): 288-289.

[17] Sun X, Li H, Wang J, Yan S. Macromolecules 2006; 39(25): 8720-8726.

[18] Sun B, Qin Y, Xu Y, Sun Y, Wang B, Dai K, Zheng G, Liu C, Chen J. Journal of Materials Science 2013; 48(15): 5354-5360.

[19] Kumaraswamy G, Verma RK, Kornfield JA. Review of scientific instruments 1999; 70(4): 2097-2104.

[20] Peterlin A. Colloid and polymer science 1987; 265(5): 357-382.

[21] Somani RH, Yang L, Zhu L, Hsiao BS. Polymer 2005; 46(20): 8587-8623.

[22] Tribout C, Monasse B, Haudin JM. Colloid and Polymer Science 1996; 274(3): 197-208.

[23] Nogales A, Hsiao BS, Somani RH, Srinivas S, Tsou AH, Balta-Calleja FJ, Ezquerra TA. Polymer 2001; 42(12): 5247-5256.

[24] Varga J. Journal of Materials Science 1992; 27(10): 2557-2579. 
[25] Somani RH, Hsiao BS, Nogales A, Srinivas S, Tsou AH, Sics I, Balta-Calleja FJ, Ezquerra TA. Macromolecules 2000; 33(25): 9385-9394.

[26] Somani RH, Hsiao BS, Nogales A, Fruitwala H, Srinivas S, Tsou AH. Macromolecules 2001; 34(17): 5902-5909.

[27] Somani RH, Yang L, Hsiao BS, Agarwal PK, Fruitwala HA, Tsou AH. Macromolecules 2002; 35(24): 9096-9104.

[28] Somani RH, Yang L, Hsiao BS. Physica A: Statistical Mechanics and its Applications 2002; 304(1): $145-157$.

[29] Agarwal PK, Somani RH, Weng WQ, Mehta A, Yang L, Ran SF, Liu LZ, Hsiao BS. Macromolecules 2003; 36(14): 5226-5235.

[30] Yang L, Somani RH, Sics I, Hsiao BS, Kolb R, Fruitwala H, Ong C. Macromolecules 2004; 37(13): 4845-4859.

[31] Somani RH, Yang L, Hsiao BS, Sun T, Pogodina NV, Lustiger A. Macromolecules 2005; 38(4): 1244-1255.

[32] Kumaraswamy G, Kornfield JA, Yeh F, Hsiao BS. Macromolecules 2002; 35(5): 1762-1769.

[33] Pogodina NV, Winter HH. Macromolecules 1998; 31(23): 8164-8172.

[34] Pogodina N, Siddiquee S, Van Egmond J, Winter H. Macromolecules 1999; 32(4): 1167-1174.

[35] Seki M, Thurman DW, Oberhauser JP, Kornfield JA. Macromolecules 2002; 35(7): 2583-2594.

[36] Leugering HJ, Kirsch G. Die Angewandte Makromolekulare Chemie 1973; 33(1): 17-23. 
[37] Devaux E, Chabert B. Polymer communications 1991; 32(15): 464-468.

[38] Varga J, Ehrenstein GW. Polymer 1996; 37(26): 5959-5963.

[39] Varga J. Journal of Macromolecular Science Part B 2002; 41(4-6): 1121-1171.

[40] Pan Y, Shi S, Xu W, Zheng G, Dai K, Liu C, Chen J, Shen C. Journal of Materials Science 2014; 49(3): 1041-1048.

[41] Liang Y, Liu S, Dai K, Wang B, Shao C, Zhang Q, Wang S, Zheng G, Liu C, Chen J. Colloid and Polymer Science 2012; 290(12): 1157-1164.

[42] Lamberti G, Brucato V. Journal of Polymer Science Part B: Polymer Physics 2003; 41(9): 998-1008.

[43] Natta G, Corrasini P. Nuovo Cimento Supplemento 1960;15(1): 40-51.

[44] Liu X, Dai K, Hao X, Zheng G, Liu C, Schubert DW, Shen C. Industrial \& Engineering Chemistry Research 2013; 52(34): 11996-12002.

[45] Liu Z, Liu X, Zheng G, Dai K, Liu C, Shen C. Journal of Materials Science 2015; 50(2): 599-604.

[46] Turner-Jones A, Aizlewood JM, Beckett DR. Macromoleculecules Chemical Physics 1964; 75(1): 134-158.

[47] Picken SJ, Aerts J, Visser R, Northolt MG. Macromolecules 1990; 23(16): 3849-3854.

[48] Ma Z, Shao C, Wang X, Zhao B, Li X, An H, Li L. Polymer 2009; 50(12): 2706-2715. 


\section{Graphical Abstract}

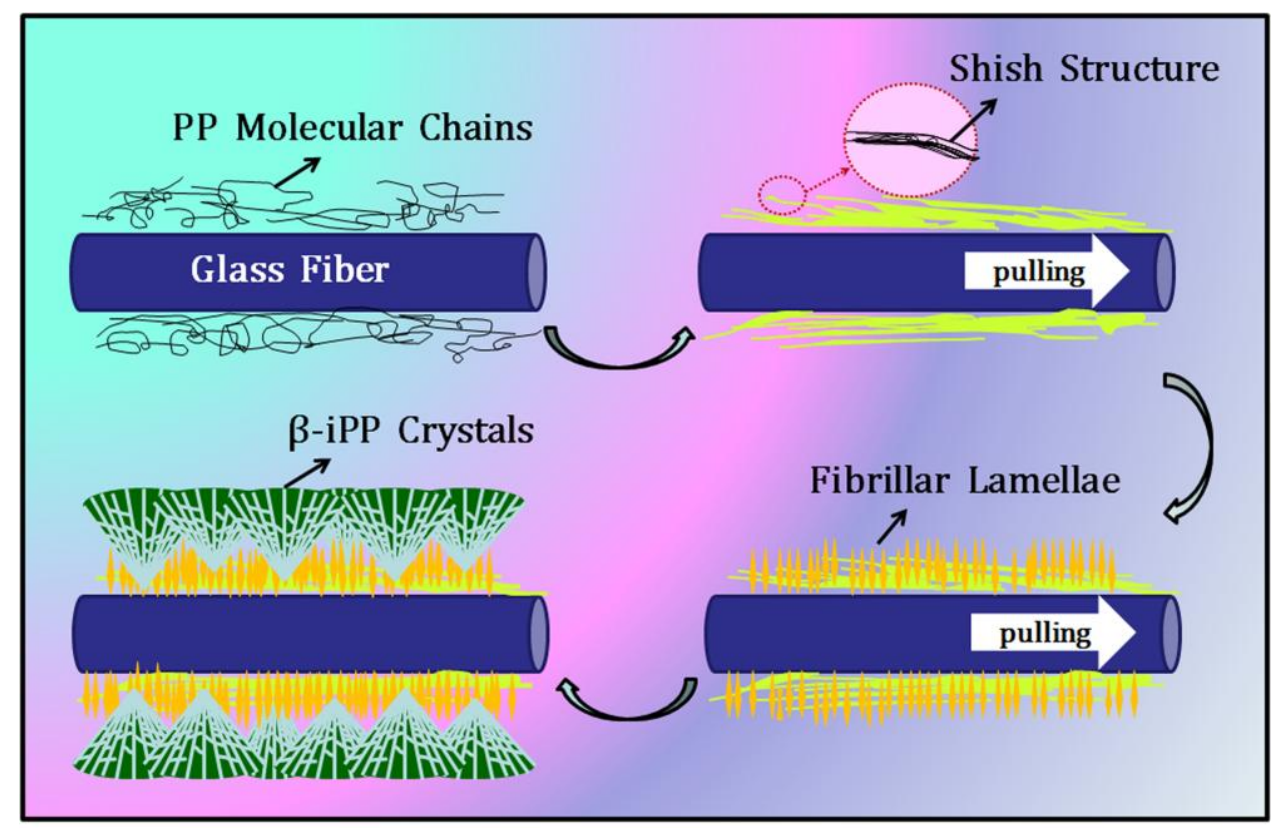

